\title{
Bullous Dermatoses in Pregnancy
}

\author{
Sarolta Kárpáti
}

Published online: 9 October 2012

(C) Springer Science+Business Media New York 2012 treatment in gravidas. This paper reviews the clinics and therapy of some unique constellations: the course of autoimmune blistering diseases during gravidity, their diagnosis, including differential from other gestational dermatoses and the possible fetal outcomes. As pregnancy induced autoimmune blistering, pemphigoid gestationis will be detailed, together with the significant development on fetal-maternal immunology related to disease pathology. Management of patients with pemphigus and pemphigoid group of diseases as well as therapy, diet, and follow-up suggested for gravidas with dermatitis herpetiformis will be summarized. In this context, the association of bullous dermatoses with further autoimmune diseases also is overviewed. Two still unclear problems will be updated: the immunology of healthy pregnancy and the fetal-maternal tolerance, including the consequences of lost or damaged tolerance during early or late pregnancy and the current status and pregnancy safety categories of immunosuppressive treatments.

\section{Immunology of Healthy Pregnancy: The Feto-Maternal Tolerance}

Gravidities represent an immunologically exceptional tolerance: the embryo and later the fetus with the maternally and paternally inherited alleles must coexist with an active semiallogeneic maternal immune system until delivery. Although the mother and the developing embryo or fetus are genetically and immunologically different, a well-regulated immunological homeostasis will ensure the healthy fetal development and the uneventful outcome of pregnancy.

The placenta has two major components: a complex vascular network system inside, covered by a complex villous epithelial system outside [1]. The internal vasculature and stroma allow and support the maternal circulation to the fetus, and the components of this complex system are 
derived from the embryonic mesoderm. The outer epithelium is an important structural and functional unit of the placenta and is built from fetal-derived trophoblast cells, which form a special fetal-maternal interface providing oxygen and nutrients to the fetus and a permanent serum exchange between mother and child.

The mobile, extravillous fetal cytotrophoblast cells of the fetal-placenta unit contact with the maternal tissues and also with the maternal immune cells; they invade the placental matrix and endothelium and meet the maternal dendritic macrophages, NK cells, and T cells [2, 3]. The classical allogeneic rejection of the fetal cells is avoided mostly because these cytotrophoblasts are MHC class II negative. Further immunological factors support the fetal tolerance at the maternal-fetal interface, such as the presence of complement inhibitory receptors, absence of major MHC expression together with expression of nonpolymorphic nonclassical presenting molecules on the extravillous fetal cytotrophoblasts in an unusual combination (HLA-G, HLA C, HLA-E) [4], as well as a special cytokine balance [3].

In autoimmune blistering diseases developing under pregnancy, it is of pathological importance that many paternal antigens, such as desmosomal, hemidesmosomal, or basement membrane proteins, also are expressed in syncytial and cytotrophoblastic cells of normal placenta and in the epithelial cells of amniotic membrane. Ultrastructurally the desmosomes, hemidesmosomes, and the basement membrane itself are very similar in the skin and amniotic epithelium. Furthermore, confirmed by immunoblotting, the amnion tissue contained desmogleins 1 and 3, desmocollins 1 and 2, desmoplakins 1 and 2, three subunits (alpha3, beta3, and gamma2) of laminin 332, bullosus pemphigoid antigen 1 (BPAg $1=$ $\mathrm{BP} 180$ ) and BPAg2 (BP230), the 97-kDa linearis IgA dermatosis (LAD) antigen and type VII collagen [5, 6]. Full-length BP180 was detected by Western immunoblot or by flow cytometry and indirect immunofluorescence (IF) in placental and cytotrophoblast cell lysates and cell cultures [7].

\section{Loss or Damage of the Feto-Maternal Tolerance in Early or Late Pregnancies}

The intimate immune contact between mother and fetus coexists with an appropriate allorecognition and tolerance at the maternal-fetal interface borders supporting a successful pregnancy outcome. Any harm or changes to this harmonic immune balance might induce immune dysregulation.

- Disorders occur in part due to immune misregulation leading to repeated miscarriages or preterm delivery without skin diseases $[8,9]$.

- Unexpected immunological events or constellations might induce pregnancy-related cutaneous immunological or autoimmune diseases, such as impetigo herpetiformis (IH) and pemphigoid gestationis (PG).

- Already existing cutaneous or systemic autoimmune diseases represent a challenge to the fetal tolerance and the disease itself also might have a different course during pregnancy [9].

Diseases primarily associated with a Th2-immune response, such as lupus erythematosus, characteristically deteriorate during pregnancy and improve after delivery. This is thought to be due to a temporary imbalance that develops between the differential cytokine secretion profiles and due to the preferentially Th1 or Th2 mediated immune responses in pregnancy preventing the rejection of the fetus. During the early phase of pregnancy, successful implantation occurs under a dominating Th1-type response in a proinflammatory microenvironment, whereas later the immunomodulation is shifted to a Th2 dominance to control endocrine and immune interactions in the advanced pregnancy [3].

\section{Pemphigoid Group of Diseases in Pregnancy}

Young adult ages are usually not characteristic for BP development, and children with juvenile BP might get into remission in childhood or before adolescence. Therefore, the majority of the rare cases with basement membrane autoimmunities in pregnancy are patients with pemphigoid gestationis (PG). There are a few reports on IgA pemphigoid and exceptionally on epidermolysis bullosa acquisita too.

\section{Pemphigoid Gestationis}

PG is a rare gestational dermatosis, an autoimmune blistering disease developing usually in the second or third trimester of pregnancy, followed by spontaneous resolution of skin symptoms after delivery. The disease, previously known as herpes gestationis, is now classified upon the immunologic and histologic features as a member of the pemphigoid group of diseases, more exactly as a unique manifestation of bullous pemphigoid (BP) [10-12]. PG similarly to BP is characterized by subepidermal blister formation and by autoantibodies targeting the hemidesmosomal proteins collagen XVII $(=\mathrm{BP} 180=\mathrm{BPAg} 1)$ and less commonly also BP230 [12-14].

Epidemiology and Clinical Features PG is a rare disease with an incidence approximately 1 case per 50,000 pregnancies. It starts in the third trimester but less commonly also during the second or first trimester or postpartum [11, 15-17]. PG is characterized by spreading itchy, erythematous, urticariform, exudative plaques, vesicles at the beginning, rarely also by vesicles or tense blisters on the 
extremities, trunk, and on the abdominal region (Table 1). During a progressive disease course, more and more blisters develop. Characteristically the periumbilical region is involved (Fig. 1). The most common differential is the itchy polymorphic eruption of pregnancy, with very similar dynamic and skin symptoms, which first appear along the abdominal striae and have no or less blistering [10, 11], but in these patients the close periumbilical skin is free of symptoms. An erythema exsudativum multiforme (palmoplantar and mucosal symptoms are more common, blisters are rare here), an impetigo herpetiformis (pustules and blisters), or even a spreading microbial eczema also should be excluded (mostly 1-2 mm vesiculopapules, erosions, partially grouped).

Pregnancy Outcome A study on 87 PG patients with 272 pregnancies reported 142 with PG complicated gravidities (approximately $50 \%$ ). The mean age of patients was 28 years, onset of PG ranged from 5 weeks gestation to 35 days postpartum [17]. Of 117 PG with detailed course, the disease developed in $17.9 \%$ during the first trimester, in $34.2 \%$ during the second trimester, in $34.2 \%$ during the last trimester, and in $13.7 \%$ during the postpartum period [17].

Diagnosis All of the other autoimmune blistering diseases (these are very rarely initiated during pregnancy, see below) should be excluded by skin histology, by direct IF as well as by indirect IF studies or enzyme-linked immunosorbent assay (ELISA) on circulating BPAg1 autoantibodies. In $\mathrm{PG}$, we find an $\operatorname{IgG}$ (IgG1, IgG3, or IgG4) staining along the basement membrane accompanied by a $\mathrm{C} 3$ deposition there. In PG the skin basement membrane (BM), IgG staining may be negative and rarely only the $\mathrm{C} 3$ can be visualized there. Even in these cases, one of the IgG subclasses can be detected along the BM.

Table 1 Clinical features of pemphigoid gestationis

\section{Heavy pruritus}

Skin symptoms

- Urticariform erythematous plaques, annular or discoid froms

- Erythematous tense blisters and crusted erosions grouped or scattered

- Multiforme-like, exsudative erythematous plaques in iris-like configuration

- Erythematous papules grouped or scattered

- Mixed picture (most commonly)

Distribution of symptoms

- Trunk and extremities

- Abdominal skin, here the periumbilical area is involved

- Palmoplantar symptoms can be present

- Mucous membranes are usually spared

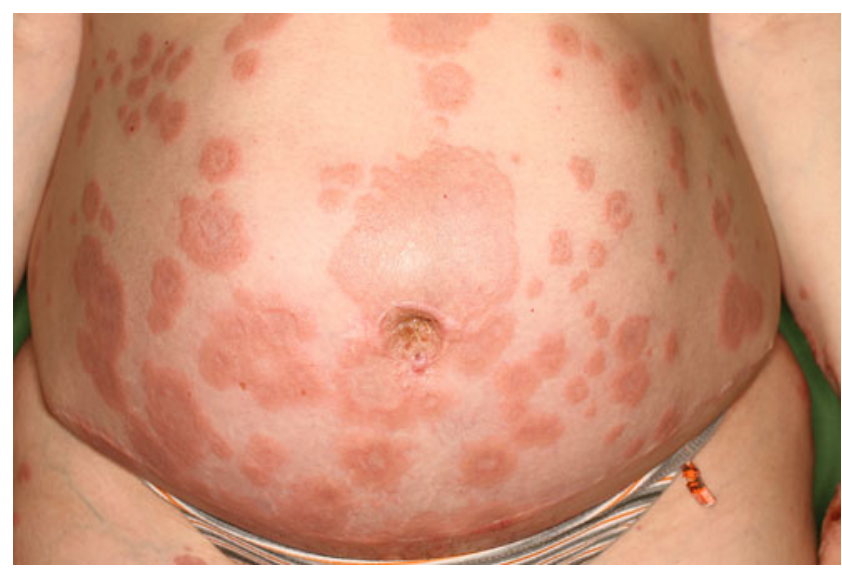

Fig. 1 Pemphigoid gestationis. Erythematous, urticariform, annular plaques on the trunk. Note the multiforme-like iris configuration and the periumbilical involvement

Associated Diseases PG can develop exceptionally in women with tumors of trophoblastic origine, such as hydatidiform mole or choriocarcinoma [18]. Here the antigenicity of the tumors cells might induce hemidesmosomal autoimmunity. It is of interest that secondary autoimmune diseases can be associated with PG, such as autoimmune thyroiditis [19].

Hormonal Influence The progressive disease course of PG also is related to changes in the estrogen and progesterone hormone levels during pregnancy. PG also has been found to flare up after administration of oral contraceptives and during menstruation $[13,14]$. Changes in progesterone level modify the humoral and cellular immune reactions, which could explain why PG is usually characterized by an immediate postpartum flare, as levels of progesterone go back down [20,21]. The case of a 46-year-old woman also supports this relation. The patient had a 17-year history of severe postpartum PG that required high-dose prednisolone for up to 10 years after her last pregnancy, went into complete remission after a chemical oophorectomy with the luteinizing hormone-releasing hormone analogue goserelin [22].

Therapy Topical, if necessary systemic corticosteroid therapy is the first choice of treatment. An adjuvant therapy with azathioprine, cyclosporine also could be helpful, but only in severe cases [23]. These are pregnancy safety level C category drugs (Table 2). Rituximab, IVIG is a good prepartum option, but there are successful reports of immunophoresis for therapyresistant disease as well [24-27]. Postpartum started PG or PG flare ups could be treated by a wider range of immunosuppressants, such as methotrexate, in severe cases by cyclophosphamide, as well as by tetracycline or minocycline [23].

Immunopathology The clinical symptoms of PG might become initiated after the expression of BPAg 2 (collagen XVII) in the amniotic tissues during the first trimester of pregnancy. The 
Table 2 Common medications for autoimmune bullous diseases during pregnancy and postpartum. Analysis of drugs by definitions of pregnancy categories by the FDA (U.S.)*

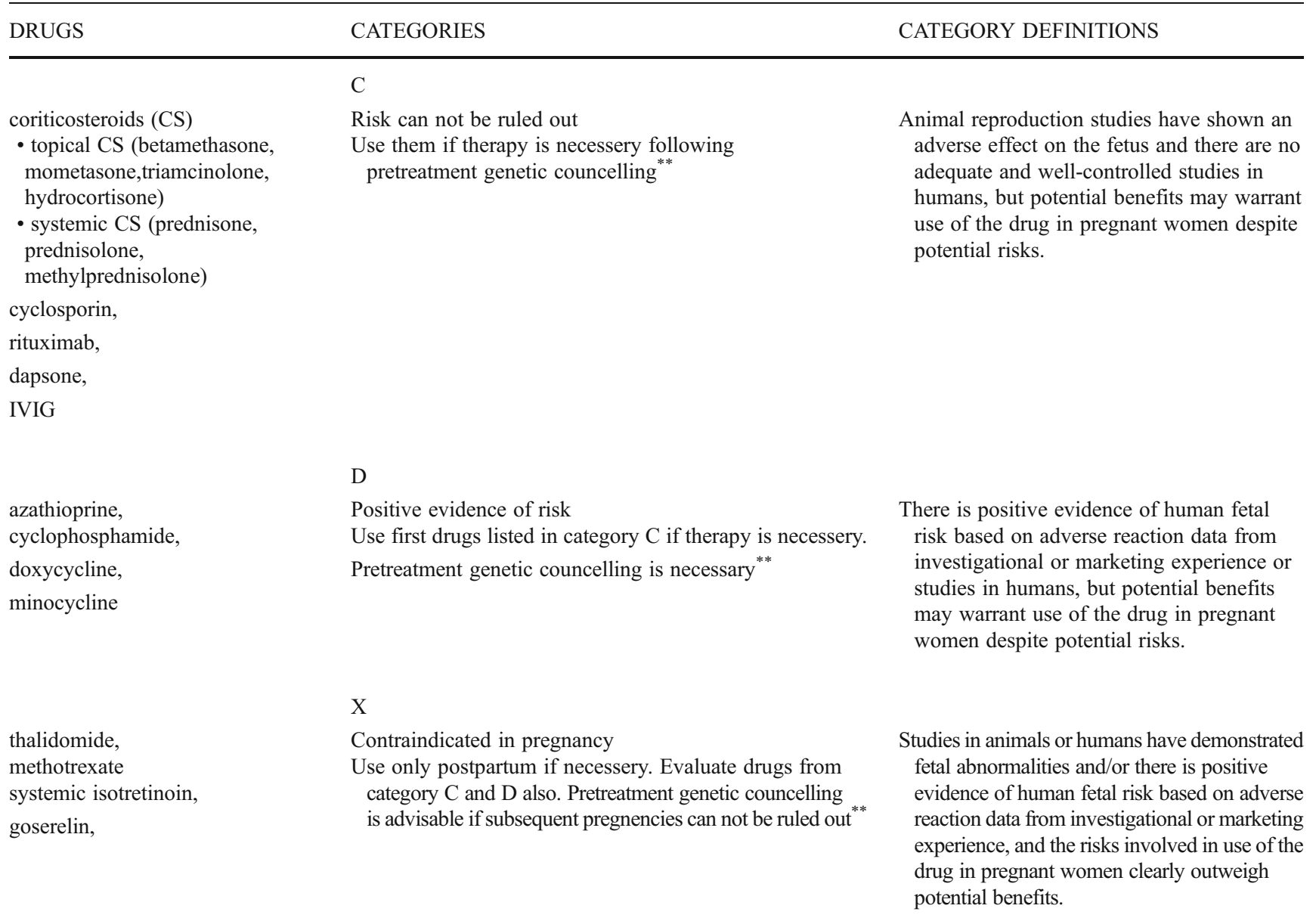

The categories are determined by the reliability of documentation and the risk to benefit ratio. They do not take into account any risks from pharmaceutical agents or their metabolites in breast milk (accessed in 2012 August at www.fda.gov/Drugs/DevelopmentApprovalProcess/DevelopmentResources/ Labeling)

* Immunoapheresis is not listed here, suggested ranking here to category C based on references avialable (author's conclusion)

***author's conclusion

histopathology of PG placentas shows mild villitis with normal collagen XVII expression levels or immunostaining pattern [7]. The BPAg 2 shed domain could contribute to the invasion of extravillous cytotrophoblasts during placental development and to the stabilization of epithelial amnion membrane [7].

In $\mathrm{PG}$, IgG-type autoantibodies are produced against the extracellular noncollagenous 16A (NC16A) domain of the $180-\mathrm{KD}$ antigen [12], which also is the immunodominant region in BP. $\operatorname{IgG} 1$ and $\operatorname{IgG} 3$ are the predominant $\mathrm{IgG}$ subclass of the disease $[11,14]$. In another paper IgG4, a subclass that can cross the placenta, was indicated as the main PG IgG in a sandwich double antibody IF study [28]. Interestingly healthy pregnant women can develop antibodies specific for BP180, and these autoantibodies are capable of binding both cyntitiotrophoblasts and placental amnion. In a large serological study on gravid and nongravid women, $\mathrm{IgE}$ but not $\mathrm{IgG}$ antibodies reactive against the NC16A domain of BP180 were found in $7.7 \%$ pregnant women $(\mathrm{n}=299)$ and in $2.2 \%$ nonpregnant healthy controls $(n=134)$, all with normal serum $\operatorname{IgE}$ and $\operatorname{IgG}$ level [7].

The epitope profile of $\mathrm{PG}$ includes target sites on the BP180 molecule outside of the NC16A domain: IgG from PG sera bound to further intracellular and extracellular epitopes by ELISA. Epitope spreading upon exposure of the fetal BP180 molecule to the maternal immune system may account for these additional autoantigens [29].

PG is associated with the presence of maternal MHC class 2 antigens DR3 and DR4 solely or rarely in combination $[1,14]$. Further detailed studies showed an association with DRB1*0301 (DR3) and DRB1*0401/040X (DR4) in a study on 41 PG women, mostly in association with either antigen alone [30]. 
Epidermolysis Bullosa Acquisita in Pregnancy

Although we have no data on epidermolysis bullosa acquisita (EBA) development under pregnancy induced by autoantibodies against the noncollagenous terminus of the $\alpha$ chain of type VII collagen, recently a newborn has been published with transient EBA due to the passive transfer of maternal autoantibodies [31]. Both the mother and her daughter had circulating autoantibodies to type VII collagen, and the newborn girl had tense blisters and erosions since birth. The mother had had suffered from EBA, and the newborn became free of symptoms without treatment. Similarly to BPAg1, VII collagen also is present in the placenta [5].

\section{IgA Pemphigoid in Pregnancy}

More patients with IgA pemphigoid (linear IgA dermatosis, $\mathrm{LAD}$ ) are young and have a long-lasting disease; however, there are not too many data about the pregnancy outcome. In a study including IgA pemphigoid 12 patients with a total of 19 pregnancies, the disease improved during pregnancy in all of them, enabling therapy to be reduced or stopped. Dapsone was taken by patients during 11 pregnancies without adverse effects. Fetal outcome was unaffected. One newborn had a single transient bulla. Most patients had a relapse approximately 3 months postpartum, even if they had previously been in remission. In two patients, disease started within 3 months of delivery. The authors found no contraindication to pregnancy in patients with $\operatorname{IgA}$ pemphigoid and concluded that therapy can be reduced or stopped whenever possible during pregnancy, but a postpartum relapse is highly possible [32].

\section{Pemphigus Diseases in Pregnancy}

Pemphigus is desmosomal autoimmunity, with two major types of the disease, pemphigus foliaceus (PF) and pemphigus vulgaris (PV) [33]. There are two possibilities of this very rare constellation development: 1) a mother with active pemphigus will be pregnant [34]; 2) pemphigus is induced under pregnancy [35]. Due to its rarity, reports on coincidence of pemphigus and pregnancy are limited to case reports and small case series, which have been recently reviewed [36]. In this overview on 49 pregnant women with immunologically proven PV, in 40 patients were the clinical data available: 33 had active disease and 7 were disease-free at the beginning of pregnancy. Thirty-seven of 49 patients ( $75 \%$ ) received prednisone in doses ranging from 5 to $300 \mathrm{mg} /$ day (mean $152.5 \mathrm{mg}$ ). Adjuvant therapies included azathioprine in five and dapsone, plasmapheresis, and plasma exchange in one patient each. Of the 44 live births, 20 (45\%) neonates had PV lesions at birth and $24(55 \%)$ were lesion-free. In all neonates,
PV lesions resolved within 4 weeks, either spontaneously or with mild topical corticosteroids treatment. Of the five intrauterine deaths, one was due to umbilical cord prolapse, one due to placental dysfunction, and one to cytomegalovirus pneumonitis. In two, the cause remained unclear. One neonate died 2 days after delivery due to meconium aspiration syndrome. Thus, the aggregate perinatal mortality rate was $12 \%$ (6/49). The postpartum course exceptionally can be refractor or persistent in pregnancy induced PV: a mucosal-dominant $\mathrm{PV}$, the gingival lesions after delivery remained unresponsive to different immunosuppressive therapies including corticosteroid and cyclophosphamide [36].

It is of interest, that recent studies support a linkage of pemphigus development to the HLA-G, a nonclassical MHC-I antigen, which has also a role in the fetal-maternal immunotolerance during pregnancy [37]. Pemphigus complicated pregnancy may have an uneventful course, in severe cases the abortion rate is considerable, and a careful monitoring of the high-risk mother and fetus is inevitable.

\section{Dermatitis Herpetiformis in Pregnancy}

Dermatitis herpetiformis (DH) is a chronic, pruritic, polymorphic skin disease within the group of autoimmune blistering dermatoses that develops mostly in patients with latent or silent gluten-sensitive enteropathy (GSE) and therefore it is considered as a gluten-induced skin disease [38]. The prevalence of DH was 75.3 per 100,000 , which is eight times lower than the prevalence of coeliac disease [39]. DH should be verified by lesional skin histology (subepidermal blister formation with papillary and intravesicular neutrophil accumulation), by perilesional direct IF (granular IgA deposition at the tips of the papillary dermis), and by serological studies for IgA transglutaminase 2 (TG2) antibodies present in the majority of DH cases. If possible to perform, the results of IgA type anti-TG3 or antideamidated gliadin-related peptide serology could be informative, particularly in TG2 seronegative cases [40]. Clinical symptoms are milder than those in pemphigus or pemphigoid, and DH has a usually benign course during pregnancy.

However, there are some unique aspects of care. 1) The most advisable treatment for $\mathrm{DH}$ is a life-long gluten-free diet (GFD), which is healthy [41]; patients can eat all fruits and vegetables, and milk and meat proteins. If the patient is not under GFD, it is strongly suggested to begin with that long before the planned gravidity, and the diet should be kept during pregnancy. (It also is necessary to go continue the GFD.) 2) DH, similar to GSE, can be associated with other autoimmune disease, such as thyroiditis, pernicious anemia, type I diabetes, and lupus erythematosus, and the pregnant patient should be checked for these diseases at least at the beginning of the pregnancy and one should be 
aware of rare initiation or flare up of the symptoms during pregnancy. 3) In DH, like celiac disease, patients without GFD, malabsorption is common, although usually milder than that in manifest GSE. Pregnant DH patients therefore should be carefully checked and be followed for mineral or vitamin deficiencies, periodontal diseases, progressive caries, dental enamel defects, and iron deficiency during pregnancy, and appropriate supplementation might be necessary. Early osteoporosis might start if malabsorption is severe. Regular dental care in pregnant DH patients is of particular importance. 4) In GSE, rarely neurological symptoms, depression, gluten-induced cerebellar ataxia, and seizures can be demonstrated. The GFD also could be important to prevent pregnancy-associated depression. 5) An association between GSE, fertility, and pregnancy outcome has been repeatedly confirmed [42].

\section{Celiac Disease and Pregnancy}

Although similar data are not available for $\mathrm{DH}$, the observations in $\mathrm{CD}$ can be extrapolated to the mild GSE forms in DH as well. A literature review between 1966 and 2000 concluded that patients with untreated CD have higher miscarriage rates, increased fetal growth restriction and lower birth weights due to the GSE and associated malabsorption. Under GFD decreased miscarriage rates, improved fetal nutritional support and overall perinatal outcome is expected in $\mathrm{CD}$. Women with unexplained infertility are at increased risk for having undiagnosed $\mathrm{CD}$, which may be a potentially modifiable (and treatable) risk factor. A prospective cohort study was performed at an academic infertility clinic in the United States. The overall prevalence of $\mathrm{CD}$ in this population was $2.1 \%(4 / 188)$. There was a significantly increased prevalence $(5.9 \%)$ of undiagnosed $\mathrm{CD}$ among women presenting with unexplained infertility $(n=51)$ [43]. In a population-based, case-control study between 1973 and 2008 , the risk of CD had been analyzed in 11,749 offspring with adverse fetal events and in 53,887 age- and sexmatched controls from the general population (Swedish Medical Birth Register). Infants born small for gestational age had a $21 \%$ increased risk for CD (95\% CI, 1.09-1.35), whereas other pregnancy exposures did not increase the risk of future GSE [44]. Furthermore, delayed onset of menarche, amenorrhea, early menopause, recurrent abortions, reduced rates of pregnancy, gonadal dysfunction, altered sperm morphology and motility, reduced sexual activity, repeated miscarriages, premature delivery, impaired fetal growth with low birth weight, and abnormal placental function have been published in GSE. Adverse pregnancy outcomes are possibly related to immune-mediated mechanisms or nutrient deficiency [45]. In a multicentric, perspective, case-control study the prevalence of undiagnosed $\mathrm{CD}$ in mothers of small for gestational age (SGA) newborns was analyzed in two groups: in 284 SGA newborns' mothers and in 196 appropriate for gestational age (AGA) newborns' mothers. TG2 antibodies IgA and IgG measured in blood samples identified two new cases of CD in asymptomatic SGA mothers. The authors suggested including the TG2 to the panel of prenatal blood test [46]. 5) with interruption in untreated pregnant $\mathrm{DH}$ patients under normal diet with severe skin symptoms, although the first choice is the GFD, a dapsone treatment promptly improves the skin symptoms, as a safety level C drug (Table 2). A vitamin C supplementation in that case is inevitable. We also should be sure that hematological, hepatic, and neurological side effects of dapsone, or rarely DRESS syndrome, will not be overlooked. 6) CD is more common in DH families: the newborn should be screened at childhood when under normal diet by TG2 serology together with serum IgA level [47].

In summary, GFD before under (and after) pregnancy is strongly suggested for patients with $\mathrm{DH}$, and repeated consultation with a dentist, dietician, and gastroenterologist is necessary.

\section{Conclusions}

Management of autoimmune blistering dermatoses in pregnancy is a multidisciplinary task. The exact histologically and immunologically confirmed diagnosis, including the type of circulating autoantibodies, is basic information to therapy, is inevitable therefore missing studies or data should be completed. Medical history, data on the course of the bullous disease, the actual clinical status, and the current therapy should be exactly documented. Data concerning previous pregnancies, paternalities, and fetal outcomes also are important. Repeated consultation with gynecologist, internist, and regular general checkups are inevitable even during uneventful pregnancies. Untreated GSE in DH is an enhanced and treatable risk factor of safe pregnancy outcome. Further medical studies, such as gastroenterologist in $\mathrm{DH}$, or specialists of other associated autoimmunities or underlying diseases, can be necessary. The therapy should be delineated in close collaboration with the medical team. Genetic advice for the patient about treatment safety, including past, current, and future medication, is strongly suggested. Therapy should be kept to a minimum, and good outcomes likely depend on the collaborative efforts of the dermatologist and obstetrician.

\section{Disclosure None.}

\section{References}

1. Yip L, McCluskey J, Sinclair R. Immunological aspects of pregnancy. Clin Dermatol. 2006;24:84-7. 
2. Houser LB. Decidual macrophages and their roles at the maternalfetal interface. Yale J Biol Med. 2012;85(1):105-18.

3. Chen, SJ, Liu YL, Sytwu HK. Immunologic regulation in pregnancy: from mechanism to therapeutic strategy for immunomodulation. Clin Dev Immunol. 2012; 258391.

4. Manaster I, Goldman-Wohl D, Greenfield C, Nachmani D, Tsukerman P, Hamani Y, Yagel S, Mandelboim O. MiRNAmediated control of HLA-G expression and function. PLoS One. 2012;7(3):e33395. Epub 2012 Mar 16.

5. Oyama N, Bhogal BS, Carrington P, Gratian MJ, Black MM. Human placental amnion is a novel substrate for detecting autoantibodies in autoimmune bullous diseases by immunoblotting. $\mathrm{Br}$ J Dermatol. 2003;148(5):939-44.

6. Noe MH, Messingham KA, Brandt DS, Andrews JI, Fairley JA. Pregnant women have increased incidence of $\operatorname{IgE}$ autoantibodies reactive with the skin and placental antigen BP180 (type XVII collagen). J Reprod Immunol. 2010;85(2):198-204.

7. Huilaja L, Hurskainen T, Autio-Harmainen H, Hofmann SC, Sormunen R, Räsänen J, Ilves M, Franzke CW, BrucknerTuderman L, Tasanen K. Pemphigoid gestationis autoantigen, transmembrane collagen XVII, promotes the migration of cytotrophoblastic cells of placenta and is a structural component of fetal membranes. Matrix Biol. 2008;27(3):190-200.

8. Carp HJ, Selmi C, Shoenfeld Y. The autoimmune bases of infertility and pregnancy loss. J Autoimmun. 2012;38(2-3):J266-74.

9. Carvalheiras G, Faria R, Braga J, Vasconcelos C. Fetal outcome in autoimmune diseases. Autoimmun Rev. 2012;11(6-7):A520-30. Epub 2011 Dec 11.

10. Holmes RC, Black MM. The specific dermatoses of pregnancy. J Am Acad Dermatol. 1983;8:405-12.

11. Ambros-Rudolph CM, Müllegger RR, Vaughan-Jones SA, et al. The specific dermatoses of pregnancy revisited and reclassified: results of retrospective two-center study on 505 pregnant patients. J Am Acad Dermatol. 2006;54:395-404.

12. Guidice G, Emery DJ, Zelickson BD, et al. Bullous pemphigoid and herpes gestationis autoantibodies recognize a common noncollagenous site on the BP 180 ectodomain. J Immunol. 1995; 151:5742-50.

13. Shornick JK. Herpes gestationis. J Am Acad Dermatol. 1987;17:539-56.

14. Semkova K, Black M. Pemphigoid gestationis: current insights into pathogenesis and treatment. Eur J Obstet Gynecol Reprod Biol. 2009;145:138-44.

15. Intong LR, Murrell DF. Pemphigoid gestationis: pathogenesis and clinical features. Dermatol Clin. 2011;29(3):447-52.

16. Shornick JK, Bangert JL, Freeman RG, et al. Herpes gestationis: clinical and histologic features of twenty-eight cases. J Am Acad Dermatol. 1983;8:214-24.

17. Jenkins RE, Hern S, Black MM. Clinical features and management of 87 patients with pemphigoid gestationis. Clin Exp Dermatol. 1999;24:255-9.

18. Takatsuka Y, Komine M, Ohtsuki M. Pemphigoid gestationis with a complete hydatidiform mole. J Dermatol. 2012;39 (5):474-6.

19. Shornick JK, Black MM. Secondary autoimmune diseases in herpes gestationis (pemphigoid gestationis). J Am Acad Dermatol. 1992;26:563-6.

20. Da Silva JA. Sex hormones and glucocorticoids: Interactions with the immune system. Ann N Y Acad Sci. 1999;876:102-17. discussion $117-8$.

21. Hughes GC. Progesterone and autoimmune disease. Autoimmun Rev. 2012;11(6-7):A502-14.

22. Garvey MP, Handfield-Jones SE, Black MM. Pemphigoid gestationis-response to chemical oophorectomy with goserelin. Clin Exp Dermatol. 1992;17(6):443-5.

23. Intong LR, Murrell DF. Pemphigoid gestationis: current management. Dermatol Clin. 2011;29(4):621-8.
24. Marker M, Derfler K, Monshi B, Rappersberger K. Successful immunoapheresis of bullous autoimmune diseases: pemphigus vulgaris and pemphigoid gestationis. J Dtsch Dermatol Ges. 2011;9(1):27-31.

25. Westermann L, Hügel R, Meier M, Weichenthal M, Zillikens D, Gläser R, Schmidt E. Glucocorticosteroid-resistant pemphigoid gestationis: successful treatment with adjuvant immunoadsorption. J Dermatol. 2012;39(2):168-71.

26. Gan DC, Welsh B, Webster M. Successful treatment of a severe persistent case of pemphigoid gestationis with antepartum and postpartum intravenous immunoglobulin followed by azathioprine. Australas J Dermatol. 2012;53(1):66-9.

27. Cianchini G, Masini C, Lupi F, Corona R, De Pità O, Puddu P. Severe persistent pemphigoid gestationis: long-term remission with rituximab. Br J Dermatol. 2007;157(2):388-9.

28. Patton T, Plunkett RW, Beutner EH, et al. IgG4 as the predominant IgG subclass in pemphigoides gestationis. J Cutan Pathol. 2006;33:299-302.

29. Di Zenzo G, Calabresi V, Grosso F, et al. The intracellular and extracellular domains of BP180 antigen comprise novel epitopes targeted by pemphigoid gestationis autoantibodies. J Invest Dermatol. 2007;127:864-73.

30. Shornick JK, Jenkins RE, Artlett CM, Briggs DC, Welsh KI, Kelly SE, Garvey MP, Black MM. Class II MHC typing in pemphigoid gestationis. Clin Exp Dermatol. 1995;20(2):123-6.

31. Abrams ML, Smidt A, Benjamin L, Chen M, Woodley D, Mancini AJ. Congenital epidermolysis bullosa acquisita: vertical transfer of maternal autoantibody from mother to infant. Arch Dermatol. 2011;147(3):337-41.

32. Collier PM, Kelly SE, Wojnarowska F. Linear IgA disease and pregnancy. J Am Acad Dermatol. 1994;30:407-11.

33. Amagai M, Stanley JR. Desmoglein as a target in skin disease and beyond. J Invest Dermatol. 2012;132(3 Pt 2):776-84.

34. Gushi M, Yamamoto YI, Mine Y, Awazawa R, Nonaka K, Taira K, Asato Y, Hagiwara K, Uezato H. Neonatal pemphigus vulgaris. J Dermatol. 2008;35:529-35.

35. Bialynicki-Birula R, Dmochowski M, Maj J, Gornowicz-Porowska J. Pregnancy-triggered maternal pemphigus vulgaris with persistent gingival lesions. Acta Dermatovenerol Croat. 2011;19(3):170 5.

36. Kardos M, Levine D, Gürcan HM, Ahmed AR. Pemphigus vulgaris in pregnancy. Analysis of current data on the management and outcomes. Obstet Gynecol Surv. 2009;64:739-49.

37. Gazit E, Loewenthal R. The immunogenetics of pemphigus vulgaris. Autoimmun Rev. 2005;4:16-20.

38. Kárpáti S. An exception within the group of autoimmune blistering diseases: dermatitis herpetiformis, the gluten-sensitive dermopathy. Immunol Allergy Clin North Am. 2012;32(2):25562.

39. Salmi TT, Hervonen K, Kautiainen H, Collin P, Reunala T. Prevalence and incidence of dermatitis herpetiformis: a 40-year prospective study from Finland. Br J Dermatol. 2011;165(2):3549.

40. Sugai E, Hwang HJ, Vázquez H, Smecuol E, Niveloni S, Mazure R, Mauriño E, Aeschlimann P, Binder W, Aeschlimann D, Bai JC. New serology assays can detect gluten sensitivity among enteropathy patients seronegative for anti-tissue transglutaminase. Clin Chem. 2010;56(4):661-5.

41. Hervonen K, Alakoski A, Salmi TT, Helakorpi S, Kautiainen H, Kaukinen K, Pukkala E, Collin P, Reunala T. Reduced mortality in dermatitis herpetiformis: a population-based study of 476 patients. Br J Dermatol. 2012.[Epub ahead of print].

42. Eliakim R, Sherer DM. Celiac disease: fertility and pregnancy. Gynecol Obstet Invest. 2001;51(1):3-7.

43. Choi JM, Lebwohl B, Wang J, Lee SK, Murray JA, Sauer MV, Green PH. Increased prevalence of celiac disease in patients with 
unexplained infertility in the United States. J Reprod Med. 2011;56 (5-6):199-203.

44. Mårild K, Stephansson O, Montgomery S, Murray JA, Ludvigsson JF. Pregnancy outcome and risk of celiac disease in offspring: a nationwide case-control study. Gastroenterology. 2012;142(1):39-45.

45. Freeman HJ. Reproductive changes associated with celiac disease. World J Gastroenterol. 2010;16(46):5810-4.
46. Baldassarre ME, Laneve A, Fontana A, Manca F, Salvia G, Barcaglioni P, Cella A, Giannuzzo S, Esposito L, Capursi T, Mastrorilli C, Padovano A, Laforgia N. Usefulness of tissue transglutaminase type 2 antibodies in early pregnancy. Immunopharmacol Immunotoxicol. 2012. [Epub ahead of print]

47. Reunala $\mathrm{T}$. Incidence of familial dermatitis herpetiformis. Br J Dermatol. 1996;134(3):394-8. 